\title{
Absorbance at 280nm
}

National Cancer Institute

\section{Source}

National Cancer Institute. Absorbance at 280nm. NCI Thesaurus. Code C48170.

The quantity of $280 \mathrm{~nm}$ light that is not transmitted through a substance or solution. 